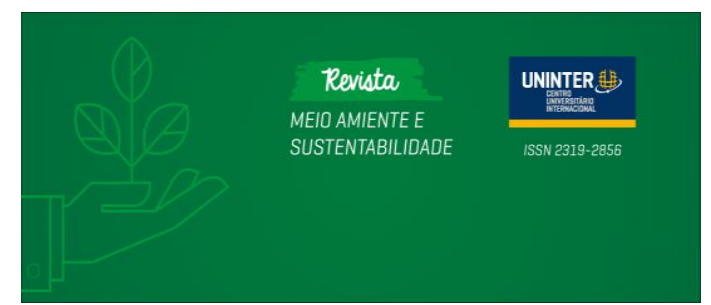

Revista Meio Ambiente e Sustentabilidade

Versão on-line ISSN 2319-2856

Volume 15, número 7. Curitiba - PR. jun/dez - 2018

\title{
AVALIAÇÃO DE IMPACTO AMBIENTAL: Análise de termos de referência para o estudo do meio biótico em licenciamentos ambientais de empreendimentos imobiliário
}

\author{
Ana Paula Lula Costa \\ anapaulalula@gmail.com
}

Universidade Federal do Paraná

\section{RESUMO}

A Avaliação de Impacto Ambiental corresponde a um documento padrão obrigatório para o Licenciamento Ambiental de atividades ou empreendimentos modificadores do meio ambiente. Apesar de toda base legislativa nacional e internacional, os estudos e relatórios de impacto ambiental ainda possuem uma série de problemas em sua execução e análise. Com isso, faz-se necessário a análise das diversas metodologias usadas para cada diagnóstico ambiental e a eficiência destas para identificação dos possíveis impactos na área. O objetivo deste estudo foi comparar diferentes termos de referências municipais de Relatórios Ambientais Prévios e elencar as metodologias usadas na avaliação do meio biológico, além de analisar seus aspectos negativos e positivos na interpretação dos impactos de empreendimentos imobiliários. Foi analisado o Termo de Referência de 3 cidades do sul do Brasil: Curitiba-PR, Novo Hamburgo-RS e Maringá-PR. Os termos de referências das diversas Avaliações de impacto se mostraram bastante divergentes e vagos em relação ao diagnóstico biológico, o que possibilita falhas metodológicas e falta de dados, o que impossibilita uma melhor análise dos possíveis impactos causados pelo empreendimento. Uma das medidas para a melhora das AIA seria, portanto, a padronização metodológica dos estudos, isto para cada bioma e grupo biológico e termos de referência que foquem em características especificas de cada região.

Palavras-chave: Relatório Ambiental, Metodologia, Diagnóstico Ambiental, Meio biótico 


\title{
EVALUATION OF ENVIRONMENTAL IMPACT: Analysis of terms of reference for the study of the biotic environment in environmental licensing of real estate projects
}

\begin{abstract}
The Environmental Impact Assessment is an obligatory document for the Environmental License for activities or enterprises that might modify the environment. Despite of all national and international guidelines, environmental impact studies and reports still have a number of problems in their implementation and analysis. Thus, it is necessary to analyze the various methodologies used in the environmental diagnosis and their efficiency identifying possible impacts in an area. The main objective of the following paper was to compare different Preliminary Environmental Reports city terms of reference and list the methodologies used in the biological elements assessment as well as its negative and positive aspects in interpreting real estate enterprise impacts. The Reference Term of 3 cities, Curitiba-PR, Novo Hamburgo-RS and Maringá-PR, in the south of Brazil, was analyzed. The terms of reference were very different and vague regarding the biological diagnosis, which leads to methodological deviations and a lack of data. Thus, it makes almost impossible to fully analyze potential impacts caused by the enterprises. One of the measures that can improve the Environmental Impact Assessment (AIA) diagnostic accuracy would be a methodological standardization of the studies, for each biome and biological group and terms of reference that indicate the specificities of each region under study.
\end{abstract}

Keywords: Environmental Report, Methodology, Environmental Diagnosis, Biotic element.

\section{INTRODUÇÃO}

A Avaliação de Impacto Ambiental, AIA, definida na Resolução do CONAMA n 1 de 1986, corresponde a um documento padrão obrigatório para o Licenciamento Ambiental de atividades ou empreendimentos modificadores do meio ambiente. O AIA visa estabelecer quais são os impactos diretos e indiretos das atividades humanas, assim como as medidas de mitigação e compensação necessárias. Neste caso, o conceito de impacto entra como limitante no processo (BRASIL, 1986). De acordo com a Resolução CONAMA 1/86, impacto ambiental é:

(...) qualquer alteração das propriedades físicas, químicas e biológicas do meio ambiente, causada por qualquer forma de matéria ou energia resultante das atividades humanas que, direta ou indiretamente, afetam: I - a saúde, a segurança e o bem-estar da população; II - as atividades sociais e econômicas; III - a biota; IV - as condições estéticas e sanitárias do meio ambiente; V - a qualidade dos recursos ambientais(...) (BRASIL, 1986).

Assim como o direito ambiental em si, a necessidade da avaliação dos impactos ambientais das atividades humanas surgiu após uma sucessão de desastres ambientais 
sofridos em decorrência ao desenvolvimento industrial. Desde então, várias conferências globais frisaram a importância da análise ambiental dos empreendimentos. No Brasil, os primeiros estudos de impacto foram impostos pelo Banco Mundial, como critério para o financiamento dos empreendimentos (SÁNCHEZ, 2013). Porém foi somente em 1981, com a Lei n 6938, que estabelece a Política Nacional do Meio Ambiente, que a AIA foi institucionalizada como um dos instrumentos de regulação ambiental (BRASIL, 1981).

Desde então a AIA ganhou vários documentos base, que se diferenciam quanto aos critérios exigidos no licenciamento ambiental de cada tipo de empreendimento, i.e. EIA/RIMA, RAP, PCA, PBA, PRAD. Essa variedade, apesar de necessária, causa alguns conflitos quanto a melhor metodologia a ser empregada em cada estudo. A legislação que discrimina cada documento tende a ser bastante ampla quanto aos aspectos exigidos, deixando ao encargo dos técnicos avaliar a autenticidade dos documentos (ALMEIDA, 2010).

A metodologia do AIA compreende primeiramente o Diagnóstico ambiental, que caracteriza a situação do meio físico, biológico e socioeconômico da área, antes da implementação do empreendimento no local; a segunda etapa analisa os impactos ambientais, quanto a sua natureza (positivo ou negativo), forma (direto ou indireto), duração (temporário ou permanente), abrangência (local ou abrangente), propriedades cumulativas e sinergéticas (ação somática); magnitude (alta, média, baixa ou irrelevante), importância e probabilidade de ocorrência. A terceira etapa deve compreender as medidas mitigatórias para os impactos negativos relevantes e um programa de monitoramento com uma série de fatores e parâmetros a serem analisados. É importante frisar que todo e qualquer estudo de impacto ambiental deve ser feito antes do início da construção ou abertura do empreendimento, sendo usado como base para o licenciamento prévio da atividade na área (BRASIL, 1986; GARCIA, 2014).

No aspecto da avaliação do meio biológico, vários estudos têm analisado metodologias que melhor caracterizam os impactos de cada empreendimento (DUARTE, DIBO E SANCHEZ, 2017). No sentido legal, a Resolução Conama 1/1986 estabelece como mínimo a caracterização da fauna e a flora, com destaque nas espécies raras, ameaçadas de extinção e indicadoras de qualidade ambiental, além das áreas de preservação permanente que possam sofrer impactos diretos ou indiretos (BRASIL, 1986). 
Apesar de toda base legislativa nacional e internacional, os estudos e relatórios de impacto ambiental ainda possuem uma série de problemas em sua execução e análise, como por exemplo, ausência de definição de escopo metodológicos, ausência de profissionais capacitados, ausência de mecanismos que assegurem a compensação e mitigação de impactos. Dentre estes, a falta de padronização no desenho experimental e técnicas de amostragem de dados pode acarretar em uma análise incompleta dos impactos e consequentemente falha na elaboração de medidas mitigatórias e compensatórias essenciais para que haja a manutenção dos serviços ecossistêmicos no Iocal (DUARTE, DIBO E SÁNCHEZ, 2017). Com isso, faz-se necessário a análise das diversas metodologias usadas para cada diagnóstico ambiental e a eficiência destas para identificação dos possíveis impactos na área.

A partir desses fatos, este trabalho tem como objetivo comparar diversos termos de referências municipais de Relatórios Ambientais Prévios e elencar as diferenças relacionadas metodologias usadas na avaliação do meio biológico e a partir disto analisar seus aspectos negativos e positivos para interpretação de impactos de empreendimentos imobiliários.

\section{FUNDAMENTAÇÃO TEÓRICA}

Licenciamento Ambiental de empreendimentos imobiliários:

O licenciamento ambiental de empreendimentos, segundo a resolução CONAMA 237/1997, deve ser competência do município no caso de impactos locais (abrange somente as dependências do município em questão) sendo que empreendimentos localizados em mais de um município, de um estado ou que estiverem alocados em unidades de conservação estadual e/ou nacional serão de competência dos órgãos ambientais estaduais e nacionais.

Muitas são as atividades sujeitas ao licenciamento ambiental, e cada qual pode requerer um tipo de estudo ambiental diferente. No caso dos empreendimentos imobiliários, as atividades que se encaixam são: o Parcelamento do Solo Urbano para fins habitacionais, industriais ou comerciais; os Loteamentos; a implantação de Conjuntos 
Habitacionais; a Construção ou Obras Civis localizadas no Litoral Paran aense; a implantação de Cemitérios; a implantação de Empreendimentos de Lazer, tais como: campings, clubes de campo, e outros.

As resoluções estaduais e municipais delimitam um pouco mais os critérios técnicos de cada estudo. No caso de empreendimentos imobiliários, tema deste estudo, a avaliação de impacto é feita pelo PCA (Plano de Controle Ambiental) e o RAP (Relatório Ambiental Prévio). O que muda primordialmente entre tais estudos é o enfoque dado a mitigação e controle de impactos, sendo que o diagnóstico ambiental se mantém o mesmo. No paraná, o IAP (Instituto Ambiental do Paraná) delimita pela Resolução SEMA 031/1998 o que deve contar no Licenciamento Ambiental de cada empreendimento. A partir deste ponto cabe ao município estabelecer os critérios específicos a serem analisado em cada avaliação ambiental, isto por meio do termo de referência para cada estudo de impacto ambiental que possa ser feito localmente.

A ideia de estabelecer um melhor planejamento urbano e gestão de novos loteamentos começou a partir da década de 70, com o surgimento do Conselho Nacional de Desenvolvimento Urbano (CNDU), que começa a regulamentar o gestão urbana e constituir o Plano Diretor de cidades com mais de 20 mil habitantes. Com a elaboração da Constituição em 1988 foi estipulado as normas de ordem pública para o uso e parcelamento de solo urbano e a necessidade da avaliação de impactos ambientais e de vizinhança para construção de novos loteamentos (ROCHA, 2012).

Entre os diversos impactos gerados pelos loteamentos urbanos temos primeiramente o desenvolviemnto das cidades, com novas vias públicas, além de edificações residenciais e comerciais. Com isso partimos para os imapctos ambientais que tais empreendimentos podem ocasionar, como o desmatamento, impermeabilização do solo, assoreamento e poluição dos rios (ROCHA, 2012; PINTO \& CHAMMA, 2013).

Todas essas situações negativas ao equilibrio ecológico do ambiente podem ser prevenidas caso haja um correto planejamento e gestão do desenvovimento urbano. Isto desde o plano diretor, onde são escolhidas as áreas prioritárias para conservação e os melhores locais a serem loteados, até o licenciamento dos empreendimentos, com um estudo efetivo dos impactos ambientais locais e as medidas mitigatórias necessárias para prevenir tais impactos. 
O principal problema das avaliações de impacto está no escopo do relatório, uma vez que relatório mal planejado não estabelece conteúdo suficiente para uma análise de impactos. Devido a isso é requerido que os orgãos responsáveis pela avalição dos estudos redijam termos de referência que deem base de apoio para pesquisas mais robustas, além de padronizar a obtenção de informações, tornando a comparação dos diferentes relatórios mais satisfatórias (ROCHA, 2012; DUARTE ET AL., 2017).

A necessidade do termo de referência se faz ainda maior no caso do diagnóstico ambiental, já que sem uma metodologia bem estruturada sempre há insuficiência na coleta e na análise dos dados, que diverge entre os relatórios . Na avaliação de impactos de loteamentos urbanos, poucas são as cidades que possuem termo de referências, e dentro das que possuem, não há uma padronização entre os processos requeridos por cada municipio (DUARTE ET AL., 2017). Com base nisso, foi analisado a metodologia para o diagnóstico do meio biótico apresentada no Termo de Referência para o RAP de 3 cidades do sul do Brasil: Curitiba-PR, Novo Hamburgo-RS e Maringá-PR.

\section{MATERIAL E MÉTODO}

Para comparar os diferentes termos de referências municipais de Relatórios Ambientais Prévios e elencar as diferentes metodologias usadas na avaliação do meio biológico foi escolhido três termos de referência usados como base em cidades localizadas na região sul do Brasil para o desenvolvimento do RAP- Relatórios Ambiental Prévio, Curitiba-PR, Novo Hamburgo-RS e Maringá-PR. Além disso, foi realizado um levantamento bibliográfico extensivo de estudos que analisam e diferenciam as principais metodologias para análise do meio biológico na avaliação de impactos ambientais- AIA, a fim de analisar seus aspectos negativos e positivos para interpretação de impactos de empreendimentos imobiliários. A busca de estudos sobre o tema foi feita em bases de dados nacionais e internacionais, como o Google Scholar, Scientific Electronic Library Online (SciELO), Research Gate e Directory of Open Access Journals (DOAJ). 


\section{RESULTADOS E DISCUSSÃO}

Os termos de referência para elaboração do RAP de empreendimentos imobiliários foram analisados somente em relação ao diagnóstico do meio biótico. Esta parte mostrou-se bastante divergente entre os três municípios analisados, isto quanto aos critérios a serem diagnosticados e a metodologia a ser utilizada. A descrição de cada termo de referência pode ser analisada na tabela 1:

TABELA 1 : Metodologia necessária para o diagnóstico do meio biótico do RAP, detalhada no termo de referência (TR) de 3 cidades do sul do Brasil: Curitiba, Paraná; Novo Hamburgo, Rio Grande do Sul e Maringá, Paraná.

\begin{tabular}{|c|c|c|}
\hline Curitiba-PR (TR 2010) & $\begin{array}{l}\text { Novo Hamburgo-RS } \\
\text { (TR 2010) }\end{array}$ & Maringá-PR (decreto 2148/2014) \\
\hline $\begin{array}{l}\text { Dentre as informações a } \\
\text { serem apresentadas deve } \\
\text { se considerar, quando } \\
\text { couber: bacia hidrográfica, } \\
\text { planta planialtimétrica da } \\
\text { área de implantação do } \\
\text { empreendimento ou } \\
\text { atividade, com locação de } \\
\text { rios, córregos, nascentes, } \\
\text { árvores isoladas com } \varnothing \text { > } \\
\text { o,15m d.a.p (diâmetro } \\
\text { o,15m superior à altura do } \\
\text { peito), bordadura de } \\
\text { bosques, áreas de } \\
\text { preservação permanente, } \\
\text { faixas não edificáveis de } \\
\text { drenagem ou outros } \\
\text { elementos presentes no } \\
\text { local, elementos da infra- } \\
\text { estrutura do entorno } \\
\text { (fornecimento de água, } \\
\text { coleta e tratamento de } \\
\text { esgoto, fornecimento de } \\
\text { gás natural, energia } \\
\text { elétrica, linhas de energia } \\
\text { elétrica, etc), inventário da } \\
\text { fauna e aspectos } \\
\text { flora, sistema } \\
\text { relacionados ao } \\
\text { viário e ao uso e ocupação }\end{array}$ & $\begin{array}{l}\text { Descrição quali- } \\
\text { quantitativa da flora } \\
\text { da área, e discorrer } \\
\text { sobre a necessidade } \\
\text { de sua remoção, se } \\
\text { houver; Descrição da } \\
\text { fauna local, incluindo } \\
\text { animais presentes na } \\
\text { área e eventuais } \\
\text { visitantes; }\end{array}$ & $\begin{array}{l}\text { Levantamento da fauna } \\
\text { considerando os principais } \\
\text { grupos existentes no lote } \\
\text { incluindo os que habitam a Área } \\
\text { de Preservação Permanente - } \\
\text { APP (se houver), suas atribuições } \\
\text { espaciais e temporais, } \\
\text { concedendo maior atenção as } \\
\text { espécies raras e ameaçadas de } \\
\text { extinção. No levantamento da } \\
\text { flora deverão ser descritas a } \\
\text { tipologia da vegetação existente, } \\
\text { conforme critérios qualitativos e } \\
\text { quantitativos, alocadas em } \\
\text { planta base (árvores com mais de } \\
\text { 15 cm de perímetro medidos na } \\
\text { altura do peito - PAP, bosques e } \\
\text { florestas), destacando as } \\
\text { espécies indicadoras de } \\
\text { qualidade ambiental. No caso do } \\
\text { lote apresentar Área de } \\
\text { Preservação Permanente - APP, o } \\
\text { responsável técnico deverá } \\
\text { declarar se a mesma está } \\
\text { regularizada ou em processo de } \\
\text { regularização, conforme as } \\
\text { diretrizes ambientais. Nos casos } \\
\text { em que a APP estiver degradada, } \\
\text { apresentar mapa em escala } \\
\text { legível indicando a(s) áreas(s) a }\end{array}$ \\
\hline
\end{tabular}




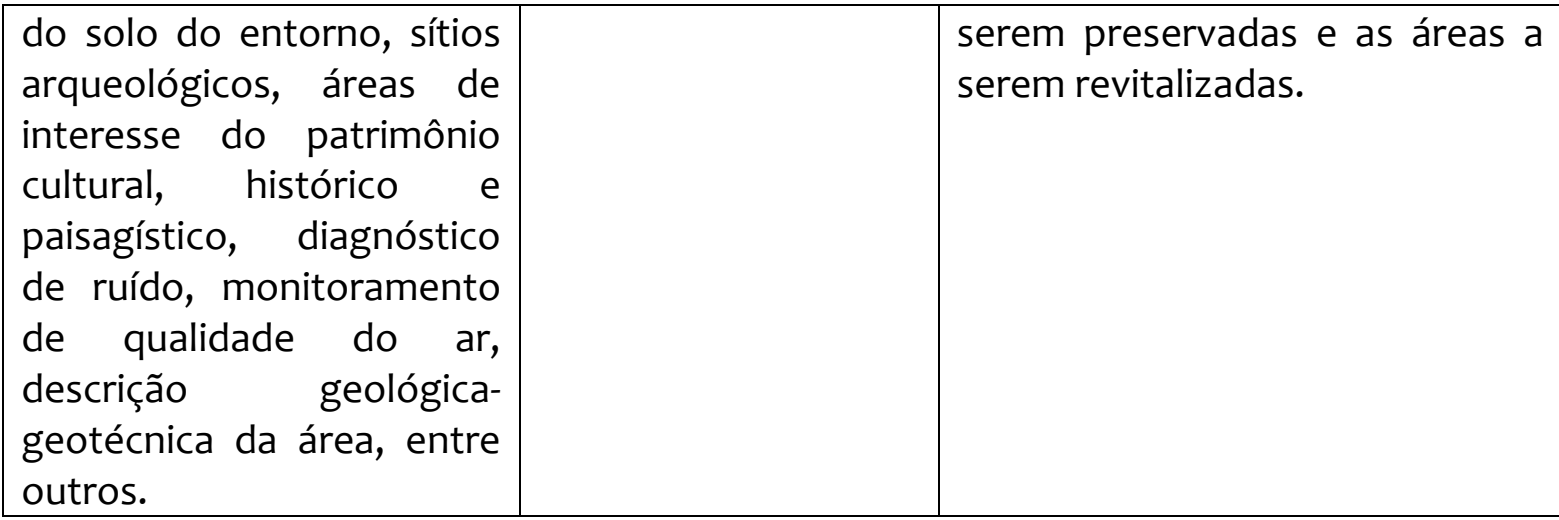

FONTE: tabela feita pelo autor, com base nos termos de referência listados. (MARINGÁ, 2014; CURITIBA, 2010; NOVO HAMBURGO, 2010).

O termo de referência da cidade de Curitiba consta uma descrição geral dos tópicos necessários para um relatório completo, porém deixa a cargo da equipe técnica as metodologias de caracterização do meio físico, biótico e sócio-econômico. Assim como a metodologia de identificação da área de influência direta e indireta, que deverá ser justificada no relatório. A única informação detalhada sobre metodologia é o paragrafo descrito na tabela, que pede para o meio biótico um inventário da fauna e flora, e o D.A.P (Diâmetro do Altura do Peito) somente de árvores isoladas. Já o termo de referência de Novo Hamburgo e o decreto estabelecido em Maringá pedem, no caso do meio biótico, uma descrição quali-quantitativa da flora e a descrição qualitativa geral da fauna. No caso de Maringá, a descrição metodológica é bem mais especifica e direciona uma metodologia padrão, apresentando critérios de seleção de espécies, "planta base (árvores com mais de $15 \mathrm{~cm}$ de perímetro medidos na altura do peito - PAP, bosques e florestas)" e o principal foco do diagnóstico "espécies raras e ameaçadas de extinção (...) destacando as espécies indicadoras de qualidade ambiental (...)".

No caso das metodologias listadas, o inventário da fauna e da flora presa pelo método qualitativo, que é a identificação de espécies de plantas e animais presentes na região de influência. Enquanto que o método quantitativo deve abranger a estrutura e tamanho populacional das espécies. A análise somente qualitativa baseia-se no levantamento biológico ou estabelece um inventário florestal da região. Ao pedir um inventário da fauna e flora da região de influência do empreendimento o TR requisita a identificação dos espécimes ou uma amostra dos indivíduos presentes na área. Em caso de áreas com maior cobertura vegetal, o inventário total é considerado pouco viável, 
devido ao seu custo benefício. O tempo que demanda a identificação de toda a flora local é demasiadamente grande, sendo que as informações obtidas com estes dados podem ser facilmente adquiridas por uma amostragem parcial da região (FREITAS \& MAGALHÃES, 2012).

Ao referir-se a fauna, temos um cenário ainda pior, o inventário faunístico total é inviável devido à dificuldade de obtenção de dados (animais não são cesseis, possuem diferentes períodos de atividade e tendem a se esconder na presença de humanos). Quanto ao levantamento da fauna da região, comumente se utiliza métodos que avaliem somente a presença/ausência de uma espécie, como busca ativa de vestígios por transectos, armadilhas e entrevista com moradores locais. Os grupos amostrados são principalmente mamíferos, aves, répteis e anfíbios (SILVEIRA, 2006).

A metodologia quali-quantitativa é importante por dar base para uma descrição fitossociologica da região, ou seja, da comunidade florística, com base em elementos como frequência, dominância e densidade das espécies vegetais (FELFILI, 2011). Para obtenção de tais dados, ligando metodologias eficientes e com bom custo beneficio é necessário fazer uma amostragem da região de estudo. A amostragem, se bem definida e planejada, pode alcançar resultados similares a um inventário total da população, além de resultar em diversos índices de sustentabilidade, dominância e equilíbrio populacional extremamente necessários para analisar os possíveis impactos que o futuro empreendimento pode causar e quais as melhores medidas de mitigação (MEDEIROS, 2004; DIAS, 2005).

Existem vários métodos de amostragem que podem ser utilizados para obtenção de dados fitossociológicos. As metodologias mais usadas para avaliações de impacto são o método de parcelas e o ponto-quadrante. Porém, além da escolha do método em si, temos os diferentes critérios de inclusão a serem estabelecidos. Estes se referem a quais grupos florísticos serão levantados e em qual tamanho/idade de crescimento. Os critérios de seleção influenciam na identificação dos padrões fitossociológicos, por isso devem escolhidos com base nos objetivos elencados para cada estudo (FELFILI, 2011). No caso dos termos de referência analisados, somente o decreto estabelecido por Maringá impõe critérios específicos para a análise florística (CAP acima de $15 \mathrm{~cm}$ de árvores lenhosas), quanto ao método de amostragem, não é especificado nenhum método padrão. 
No caso do levantamento faunístico, geralmente é feito uma analise qualitativa dos grupos chave da região em estudo. O método geralmente baseia-se na busca ativa por rastros e vestígios e ou observação de espécies, além do uso de armadilhas fotográficas e de captura para maior eficácia possível (TOMASULO, 2015). Para que o levantamento faunístico seja efetivo deve se englobar uma análise prévia bastante específica de cada grupo a ser levantado, como comportamento, horário de atividade, possíveis locais de esconderijo ou tocas. Porém, o grande problema do inventário de fauna encontra-se na falta de tempo hábil e no grande percentual de sub-amostragem devido a restrições metodológicas (proibição de captura e abate).

A dificuldade encontrada pelos profissionais na obtenção de dados, além do curto prazo de entrega do relatório e a falta de recursos financeiros faz com que grande parte das listas faunísticas sejam baseadas em dados secundários (outros relatórios, entrevistas com a população local ou pesquisas na região). O que não descreve o real cenário da área de estudo, inviabilizando uma análise efetiva dos possíveis impactos no local (SILVEIRA, 2006; SILVEIRA ET AL, 2010). De modo geral, o método de análise com maior robustez irá divergir bastante entre os diversos biomas de nosso país, além das diferentes paisagens que nos cercam. Algo que deveria ser pensado e instruído no termo de referência de cada estudo de impacto.

A falta de metodologia definida ou pelo menos, mais criteriosa, faz com que os estudo de impacto se mantenham em um nível básico e pouco abrangente (SÃO PAULO, 1995). Que foca somente na aprovação do relatório, com o mínimo de esforço necessário. Ao invés de encontrar o máximo de informações possíveis para uma análise profunda dos possíveis impactos que podem ser causados pelo empreendimento. De acordo com a revisão de estudos sobre AIA feita por DUARTE, DIBO E SANCHEZ (2017) mostra que a maioria das criticas feitas ao diagnóstico do meio biótico se encontra na falha metodológica e falta de critérios padrões estabelecidos para cada AIA em específico. A falha no diagnóstico acarreta na análise superficial ou até mesmo errônea dos impactos, o que pode levar a consequências desastrosas.

Os possíveis impactos relacionados a loteamentos urbanos vão desde erosões, até aumento de trafego local, diminuição de áreas verdes e perda da biodiversidade (PINTO \& CHAMMA, 2013). No caso da perda da biodiversidade o maior fator que leva a tal prejuízo 
é a fragmentação de habitats e áreas de preservação com baixa capacidade suporte, não conseguindo manter a riqueza de espécies que possui, levando a extinções locais. Tal acontecimento pode ser previsto pela ecologia de paisagens, que aborda um estudo voltado para escala espacial. A sobrevivência das espécies em um ambiente fragmentado depende de sua adaptação local e sua capacidade de migração entre áreas, a fim de aumentar suas chances de sobrevivência e reprodução (PRIMARCK \& RODRIGUES, 2011). Portanto, uma análise simples de presença e ausência não é suficiente para saber o grau de interferência de um empreendimento no equilíbrio ecológico local (KOBLITZ ET AL., 2011). Por isso deve-se ter um planejamento criterioso na metodologia do diagnóstico, tanto no desenho experimental, quanto na possível abrangência direta e indireta do empreendimento.

O diagnóstico ambiental também é extritamente necessário para as possiveis ações de manejo e monitoramento do impacto. Além de um levantamento das espécies da área, é imprecindivel determinar os processos ecológicos resultantes da interação entre as espécies do local. Isto porque a compensação deve ser feita de modo a manter não só o número de espécies, mas também os serviços ecológicos prestados por elas (KOBLITZ ET AL., 2011). Atualmente, os termos de referência possuem uma visão defasada da ideia de conservação ecológica, baseando-se na diversidade taxonomica como indicador de equilibrio ambiental. Porém, estudos sobre conservação já trabalham a ideia da diversidade funcional e filogenética, que possibilita uma visão mais abrangente sobre o ambiente, o que pode resultar em diferentes diagnósticos (VELLEND, 2017; MARTINS ET AL, 2018). A relação da ciência com os relatórios ténicos deve avançar em ambas vertentes. Tanto a ciência precisa de mais prática quanto os relatórios precisam de maior embasamento teórico (LIMA ET AL., 2010).

Segundo ALMEIDA (2010) as previsões de impactos nos estudos ainda são insatisfatórias, isso principalmente por serem estabelecidos com base em metodologias vagas e pouco abrangentes. Além disso, a valorização do inventário pela legislação dá uma ideia de listagem de espécies e não de análise categórica, além da supervalorização de grupos biológicos específicos. Uma das medidas para a melhora das AIA seria, portanto, a padronização metodológica dos estudos, isto para cada bioma e grupo biológico (SILVEIRA, 2006). 
A municipalização do licenciamento ambiental de empreendimentos imobiliários criou a oportunidade de estabelecer termos de referências mais focais e criteriosos para as regiões de estudo, além de possibilitar uma maior interação entre técnicos e avaliadores, a fim de tentar estabelecer a melhor metodologia para o cenário em que atuam. Porém, esta não é a realidade que vemos hoje. Os termos de referências das diversas Avaliações de impacto ainda são bastante vagos e não se atentam para a principal etapa do estudo, o diagnóstico. Um vez que sem este a análise de impactos seria inviável. Faz se necessário, portanto, uma maior interação do meio técnico com a pesquisa ciêntifica, de modo a estabelecer termos de referência que possuam uma maior base metodológica e priorize a obtenção de resultados que realmente corresponderão ao real estado ecológico da região estudada. Com análises mais robustas poderemos prever melhor os impactos e estabelecer ações de monitoramento e manejo com um maior custo-beneficio a todos.

\section{CONSIDERAÇÕES FINAIS}

A análise dos termos de referência mostra a grande diferença nas atribuições e critérios estabelecidos em cada cidade. Sendo que o nível de especificação dos dados e a metodologia de obtenção dos mesmos se mantém bastante baixo. A falta de padronização de critérios e elementos chave para as avaliações de impacto ambientais permite a realização de relatórios rasos, com falta de dados importantes que levarão a negligência de possíveis impactos e falhas em ações de mitigação. Além de consequências técnicas a falta de critérios leva a uma maior demora na aprovação do licenciamento ambiental, uma vez que o relatório de impacto deverá ser avaliado mais de uma vez, até que se obtenha os dados necessários para a aprovação. Um outro fator importante é que apenas um dos três termos de referência pediu o foco na análise da qualidade ambiental do local, focando para espécies bioindicadoras e espécies chave de equilíbrio ecológico. Este critério deve ser estabelecido como primordial na avaliação de impactos, uma vez que só é sabido o grau de resistência a perturbações de uma área de preservação após avaliar o nível ecológico e a qualidade ambiental em que esta se encontra. Portanto, a partir desta revisão sobre critérios e metodologias dos Relatórios 
Ambientais é possível concluir que os relatórios ambientais em nível municipal ainda são bastante rasos, com diversas brechas que impedem a eficiente avaliação de impactos. Devido a isso, é aconselhado uma revisão dos termos de referências para os RAPs e demais estudos, levando em conta aspectos gerais em nível ambiental, como grupos biológicos focais, metodologias chave e critérios mínimos para análise de impactos, e específicos de cada cidade, como o bioma em que ela se encontra, o nível de fragmentação das áreas verdes e etc.

\section{REFERÊNCIAS}

ALMEIDA, M. R. R. Análise da qualidade de relatórios de controle ambiental aprovados pela superintendência regional de meio ambiente e desenvolvimento sustentável do Sul de Minas Gerais. 2010. 154 f. Dissertação. Pós-graduação em Meio Ambiente e Recursos Hídricos, Universidade Federal de Itajubá, Minas Gerais. 2010.

BRASIL. Lei $\mathbf{n}^{\circ}$ 6.938, de 31 de agosto de 1981. Dispõe sobre a Política Nacional do Meio Ambiente, seus fins e mecanismos de formulação e aplicação, e dá outras providências. Diário Oficial da União, Brasília, 2 set. 1981.

BRASIL, Ministério do Meio Ambiente, Conselho Nacional do Meio Ambiente, CONAMA. Resolução CONAMA n 001, de 23 de janeiro de 1986. Diário Oficial da União, Brasília, 17 fev. 1986.

BRASIL, Ministério do Meio Ambiente, Conselho Nacional do Meio Ambiente, CONAMA. Resolução CONAMA n²37, de 19 de dezembro de 1997. Diário Oficial da União, Brasília, 17 fev. 1986.

CURITIBA, Prefeitura Municipal de Curitiba, Secretaria Municipal do Meio Ambiente. Termo de referência para elaboração do Relatório ambiental prévio - RAP. Curitiba, PR. 2010.

DIAS, A. C. Composição florística, fitossociologia, diversidade de espécies arbóreas e comparação de métodos de amostragem na floresta Ombrófila densa do parque estadual Carlos Botelho/SP-Brasil. 2005. 184p. Tese. Escola Superior de Agricultura Luiz de Queiroz, Piracicaba, 2005.

DUARTE, C. G.; DIBO, A. P. A.; SÁNCHEZ, L. E. (2017). O que diz a pesquisa acadêmica sobre avaliação de impacto e licenciamento ambiental no Brasil? Ambiente \& sociedade, v. 20, n.1, p. 245-278.

FELFILI, J. M.; EISENLOHR, P. V.; MELO, M. M. R. F.; ANDRADE, L. A.; NETO, J. A. A. M. Fitossociologia no Brasil: Métodos e Estudos de Casos. Vol 1. Minas Gerais: UFV, 2011.

FREITAS, W. K.; MAGALHÃES, L. M. S. (2012). Métodos e Parâmetros para Estudo da Vegetação com Ênfase no Estrato Arbóreo. Floresta E Ambiente, 19(4), 520-540. https://doi.org/10.4322/floram.2012.054. 
GARCIA, K. C. Avaliação de Impactos Ambientais. Curitiba: Intersaberes. 2014. 256p.

KOBLITZ, R. V.; JÚNIOR, S. J. P.; DE ALBUQUERQUE AJUZ, R. C.; GRELLE, C. E. V. (2011). Ecologia de paisagens e licenciamento ambiental. Natureza a Conservação: 9(2), 244-248. https://doi.org/10.4322/natcon.2011.033.

LIMA F. P.; MUNIZ J. R.; MARCO JUNIOR P. (2010) Evaluating Brazilian conservation projects: the weak link between practice and theory. Natureza \& Conservação: 8, 41-45. http://dx.doi. org/10.4322/natcon.00801006.

MARINGÁ, Prefeitura Municipal de Maringá. Resolução n 2.148, de 2014. Órgão Oficial do Município, 03 out. 2014.

MARTINS, V. F.; SEGER, G. D. D. S.; WIEGAND, T.; SANTOS, F. A. M. D. (2018). Phylogeny contributes more than site characteristics and traits to the spatial distribution pattern of tropical tree populations. Oikos. https://doi.org/10.1111/ele.13089.

MEDEIROS, D. A. Métodos de amostragem no levantamento da diversidade arbórea do cerradão da Estação Ecológica de Assis. 2004. 85 p. Dissertação (Mestrado). Escola Superior de Agricultura Luiz de Queiroz, Piracicaba, 2004.

NOVO HAMBURGO, Prefeitura Municipal de Novo Hamburgo, Secretaria municipal de meio ambiente, SEMAM. Termo de referência para elaboração do relatório ambiental prévio. Novo Hamburgo, RS. 2010.

PINTO, E. S.; CHAMMA, P V. C. (2013). Os Loteamentos Urbanos E Seus Impactos Ambientais E Territoriais : O Caso Do Loteamento Villaggio li Na Cidade De Bauru-SP. Revista Nacional de Gerenciamento de Cidades, v. 01, n. 03, pp. 95-135.

PRIMACK, R. B.; RODRIGUES, E. Biologia da conservação. 1 ed. Londrina: Editora Planta, 2011.

ROCHA, G. L. Avaliação da aplicação do estudo de impacto ambiental em projeto de loteamento urbano localizado em área de proteção de mananciais: UTP de Pinhais, UTP do Itaqui e APA de Piraquara - região metropolitana de Curitiba/PR. Dissertação. Pós Graduação em Engenharia da Construção Civil, Universidade Federal do Paraná. Curitiba, 2012.

SÁNCHEZ, L. E. Avaliação de impacto ambiental: conceitos e métodos / 2. ed. -- São Paulo: Oficina de Textos, 2013. 553p.

SÃO PAULO. Secretaria do Meio Ambiente, Coordenadoria de Planejamento Ambiental. A efetividade do processo de avaliação de impacto ambiental no estado de São Paulo: uma análise a partir de estudos de caso. São Paulo: Secretaria do Meio Ambiente, 1995.

SILVEIRA, R. L. Avaliação dos métodos de levantamento biológico terrestre em estudos de impacto ambiental para construção de usinas hidrelétricas na região do Cerrado. 2006. 66 f. Dissertação. Pós-graduação em Ecologia Aplicada, Universidade de São Paulo, São Paulo. 2006. 
SILVEIRA, L. F., BEISIEGEL, B. DE M., CURCIO, F. F., VALDUJO, P. H., DIXO, M., VERDADE, V. K., ... CUNNINGHAM, P. T. M. (2010). Para que servem os inventários de fauna? Estudos Avançados: 24 (68), 173-207. https://doi.org/10.1590/S0103-40142010000100015.

TOMASULO, P. L. B. Gestão da Biodiversidade: Uma Análise com foco na preservação ambiental. Curitiba: Intersaberes, 2015. 200p.

VELLEND, M. (2017). The biodiversity conservation paradox. American Scientist, v. 105, n. 2, p. 94. 